Sharif University of Technology
Scientia Iranica
SCIENTIA
IRAN I CA

\title{
Particle flow simulation in a channel with symmetric protuberances using combination of lattice Boltzmann and smoothed profile methods
}

\author{
A. Kouhestani ${ }^{a}$, M. Rahnama ${ }^{a, *}$, S. Jafari ${ }^{\text {b }}$ and E. Jahanshahi Javaran ${ }^{\text {c }}$ \\ a. Department of Mechanical Engineering, Shahid Bahonar University, Kerman, P.O. Box 76169133, Iran. \\ b. Department of Petroleum Engineering, Shahid Bahonar University, Kerman, Iran. \\ c. Department of Energy, Institute of Science, High Technology and Environmental Sciences, Graduate University of Advanced \\ Technology, Kerman, Iran.
}

Received 23 May 2015; received in revised form 26 April 2016; accepted 16 August 2016

\author{
KEYWORDS \\ Lattice Boltzmann \\ method; \\ Smoothed profile \\ method; \\ Stenosis; \\ Blood; \\ Particle.
}

\begin{abstract}
In the present study, a combination of Lattice Boltzmann Method (LBM) and Smoothed Profile Method (SPM) is used to simulate one, two, and many particles motion in a planar channel with two symmetric protuberances. LBM is applied as the fluid flow solver and SPM is used to satisfy the no-slip boundary condition at particles surfaces. Bounceback boundary conditions are used for lower and upper walls while pressure boundary conditions are applied for the fluid inlet and outlet boundaries. Horizontal, vertical, and angular velocities of particles are recorded during the simulation. It is concluded that the combined LBM-SPM can be considered as a good candidate for simulation of particle motion in a channel with stenotic geometry.
\end{abstract}

(C) 2017 Sharif University of Technology. All rights reserved.

\section{Introduction}

Blood is a bodily fluid that delivers necessary substances, such as nutrients and oxygen, to cells and transports metabolic waste products away from those same cells. Over the years, many experimental and theoretical investigations have been done into the subject of blood flow dynamics. These investigations can lead to better understanding of cardiovascular diseases and development of pathological patterns. Numerical analysis can improve our understanding of blood flow considering experimental evidence [1].

Particle suspension simulation through stenosis arteries is a subject of great interest in medical science [2]. A stenosis is an abnormal narrowing or contraction of a body passage or opening such as blood vessels which in turn cause reduction of blood flow and

\footnotetext{
*. Corresponding author.

E-mail address: rahnama@uk.ac.ir (M. Rahnama)
}

red blood cells. This causes health risks and mortality in humans [3].

In two recent decades, Lattice Boltzmann Method (LBM) has been used as an alternative method to Navier-Stokes equations for fluid flow simulation. The main concern in LBM simulation of particulate flows is treating the no-slip boundary condition at the fluidparticle interface. A detailed review of LBM by Aidun and Clausen [4] revealed that LBM has great success in simulation of particle suspensions in fluid flow. One of the main advantages of using LBM in such flow configurations is overcoming the limitations of the conventional finite volume and finite-element methods by using a system of fixed grid distribution. Generally, two methods are used in simulating particles suspensions in the context of LBM, namely link-based and nodebased methods. Standard bounce-back method is the simplest one in link-based methods $[5,6]$, but its main drawback lies in step-wise representation of curved and complex surfaces which results in fluctuations 
in computation of forces exerted on these surfaces. However, this drawback may be mitigated by using finer grids or interpolated bounce-back methods.

In node-based methods, a combination of the Immersed-Boundary Method (IBM) and LBM is used to simulate particle-fluid intercation [7]. In other words, in addition to the Eulerian LBM grid, a Lagrangian grid is used to track the surface of the particles. The particle boundary is treated as a deformable medium with high stiffness, and a small distortion of the particle boundary yields a force that tends to restore the particle into its original shape. As in traditional IB techniques, the force term is applied to the fluid as a force density through an appropriate weight function. In LBM, several methods are used to add a forcing term into the LB equation [8]. It should be noted that body force calculation in this method is not a straightforward task.

As mentioned before, the main issue in particle flow simulation is the application of no-slip boundary condition at the interface of fluid and solid phases, requiring local fluid and solid velocities at their interface to be the same. To satisfy this boundary condition, Nakayama and Yamamoto [9] proposed a direct forcing method called Smoothed Profile Method (SPM), which was used in their Navier-Stokes equations solver. In this method, the effect of particles on the host fluid is represented by smooth body forces. It states that Navier-Stokes equations are solved with a body force without any boundary condition at the particle-fluid interface. As will be shown in the following section, body force calculation in this method is a straightforward procedure. Considering this feature, the drawbacks of link-based methods and complicated task of body force calculation in immersed boundary methods on one hand and the advantage of LBM and SPM in using regular Cartesian grids on the other hand motivated Jafari et al. [10] to combine these methods. Recently, this method has been used to study the rheological behavior of particle suspension fluids [11].

The main objective of the present study is to develop application of SPM-LBM to stenotic geometries as a model for simplified computational hemodynamics. In order to illustrate the accuracy of this method in such types of simulations, a particle suspension in a two-dimensional stenotic geometry is simulated.

This kind of flow is of great interest in medical science. Such a problem was studied by $\mathrm{Li}$ et al. [2] via LBM. In their simulations, they employed a combination of boundary conditions proposed by Filippova and Hanel [12] for the stationary complex geometry and the Newtonian dynamics. In their work, they extended the method to simulate two-dimensional particle suspensions in symmetric stenotic arteries. Recently, Wu and Shu [13] have used the method proposed by Shu et al. [14] to satisfy the non-slip boundary condition. They mentioned that for the particulate flows, the motion of particles is controlled by the force and torque exerted on them. Therefore, it is necessary to accurately compute the hydrodynamic force acting on the boundaries of particles. In terms of accuracy in resolving flow field, it was shown that the boundary condition-enforced IB-LBM provides a simple and accurate way to compute lift and drag (vertical and horizontal) forces exerted on the immersed object [15].

In this paper, SPM-LBM is used to simulate fluid flow in two-dimensional particle suspensions in symmetric stenotic arteries. As an advantage of using this method, no additional boundary conditions, such as the bounce-back boundary condition, need to be satisfied. Section 2 describes numerical simulation method including LBM and SPM. Section 3 is devoted to simulation results. Finally, conclusions are presented in section four.

\section{Numerical method}

\subsection{Simulation of fluid flow using lattice Boltzmann method}

In the present work, the two-dimensional lattice Boltzmann method with a single-relaxation-time collision operator is applied for fluid flow simulation. The discretized form of Boltzmann equation can be written as [16]:

$$
f_{\alpha}\left(\mathbf{x}+\mathbf{e}_{\alpha} \delta t, t+\delta t\right)=f_{\alpha}(\mathbf{x}, t)-\frac{1}{\tau}\left[f_{\alpha}(\mathbf{x}, t)-f_{\alpha}^{\mathrm{eq}}(\mathbf{x}, t)\right]
$$

in which $f_{\alpha}, \mathbf{x}, \mathbf{e}_{\alpha}, t, \tau$, and $\delta t$ are the particle distribution function, position vector, discrete velocity vector, time, dimensionless relaxation time, and time step, respectively. Discrete velocity vectors in the most widely-used two-dimensional lattice model $\left(D_{2} Q_{9}\right)$ are written as follows [16]:

$$
\mathbf{e}_{\alpha}=\left\{\begin{array}{l}
(0,0) \\
\alpha=0 \\
\left(\cos \left[(\alpha-1) \frac{\pi}{2}\right], \sin \left[(\alpha-1) \frac{\pi}{2}\right]\right) c \\
\alpha=1-4 \\
\left(\cos \left[(2 \alpha-9) \frac{\pi}{4}\right], \sin \left[(2 \alpha-9) \frac{\pi}{4}\right]\right) \sqrt{2} c \\
\alpha=5-8
\end{array}\right.
$$

where $c$ is defined as $\delta x / \delta t$ and $\delta x$ is the lattice length unit. Equilibrium distribution function, $f_{\alpha}^{\mathrm{eq}}$, is chosen, such that the Navier-Stokes equations for a weakly compressible system are obtained. It is expressed as in the following equation for $\mathrm{D}_{2} \mathrm{Q}_{9}$ model:

$$
f_{\alpha}^{\mathrm{eq}}=\omega_{\alpha} \rho\left[1+\left(\mathbf{e}_{\alpha} \cdot \mathbf{u}\right) / c_{s}^{2}+\left(\mathbf{e}_{\alpha} \cdot \mathbf{u}\right)^{2} / 2 c_{s}^{4}-\mathbf{u}^{2} / 2 c_{s}^{2}\right],
$$


in which $\omega_{\alpha}$ is a weight factor which is $4 / 9$ for $\alpha=0$, $1 / 9$ for $\alpha=1-4$, and $1 / 36$ for $\alpha=5-8 ; c_{s}$ is the speed of sound and is defined by $c_{s}=\frac{c^{2}}{3} ; \rho$ and $\mathbf{u}$ are the Macroscopic fluid density and velocity, respectively, which can be calculated as follows:

$$
\begin{aligned}
& \rho=\sum_{\alpha=0}^{8} f_{\alpha}, \\
& \mathbf{u}=\frac{1}{\rho} \sum_{\alpha=1}^{8} \mathbf{e}_{\alpha} f_{\alpha} .
\end{aligned}
$$

Pressure can be obtained from the definition of sound velocity as $P=\rho c_{s}^{2}$.

\subsection{Fluid-solid interaction simulation using Smoothed Profile Method (SPM)}

In smoothed profile method, the interface between fluid and solid particle is considered to have a width in the order of grid spacing [9]. In other words, each particle surface is represented by a smoothed profile which equals unity in the particle domain, zero in the fluid domain, and varies smoothly between one and zero in the solid-fluid interfacial domain (Figure 1).

Here, field quantities, such as velocity and density, are defined on the entire computational domain, which includes not only fluid regions, but also regions in which particles exist. In order to determine regions occupied by solid particles, density field, $\phi(\mathbf{x}, t)$, is defined as:

$$
\phi(\mathbf{x}, t)=\sum_{i=1}^{N_{P}} \phi_{i}(\mathbf{x}, t),
$$

where $\phi_{i}(\mathbf{x}, t) \in[0,1]$ is the density profile of the $i$ th particle (equals 1 for the points inside the particle and zero, otherwise), and $N_{p}$ is the total number of the particles in the computational domain. Several analytical

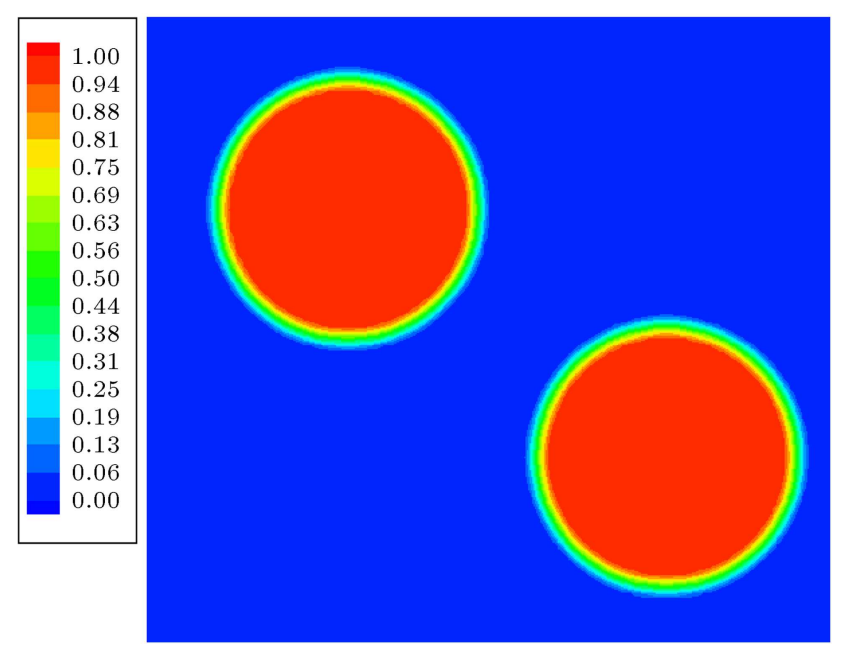

Figure 1. Schematic representation of two particles in smoothed profile method. forms of the smoothed profile for the spherical particles can be found in the literature $[9,17]$. The most widely used one is:

$$
\begin{aligned}
& \phi_{i}(\mathbf{x}, t)=s\left(R_{i}-\left|\mathbf{x}-\mathbf{R}_{i}(t)\right|\right), \\
& s(x)= \begin{cases}0 & x<-\xi / 2 \\
\frac{1}{2} \sin \left(\frac{\pi x}{\xi}+1\right) & |x| \leq \xi / 2 \\
1 & x>\xi / 2\end{cases}
\end{aligned}
$$

where $R_{i}, \mathbf{R}_{i}$, and $\xi$ are the radius, position vector, and interfacial thickness of the $i$ th particle, respectively.

Based on this concentration field, the particle velocity field is constructed from the rigid motions of $N_{p}$ particles as follows:

$$
\begin{aligned}
\phi(\mathbf{x}, t) \mathbf{u}_{P}(\mathbf{x}, t)= & \sum_{i=1}^{N_{P}} \phi_{i}(\mathbf{x}, t)\left[\mathbf{V}_{i}(t)+\boldsymbol{\omega}_{i}\right. \\
& \left.\times\left\{\mathbf{x}-\mathbf{R}_{i}(t)\right\}\right] .
\end{aligned}
$$

Here, $\mathbf{u}_{P}$ is velocity of particle, and $\left\{\mathbf{V}_{i}, \boldsymbol{\omega}_{i}\right\}(i=$ $\left.1, \cdots, N_{P}\right)$ are the translational and angular velocities of the $i$ th particle, respectively.

Virtual fluid nodes located inside solid particles and solid nodes must have the same velocities. Therefore, a body force is used inside solid particle to enforce the virtual fluid to satisfy rigid-body motion constraint. This force is zero outside of the solid domain (Figure 2).

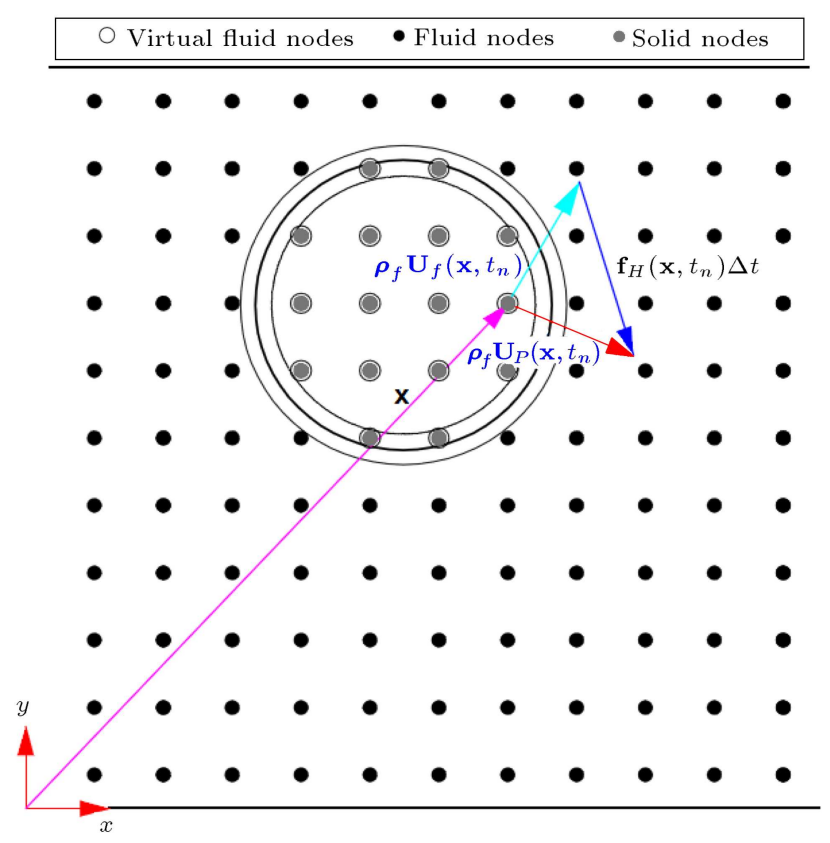

Figure 2. Solid, fluid, and virtual fluid, nodes for computation of fluid-solid interaction force, $\mathbf{f}_{H}$. 
In the smoothed profile method, lattice Boltzmann equations are solved for the entire computational domain, including both solid and fluid phases. However, the effects of solid particles on flow in the form of no-slip boundary condition at the interface of these phases and rigid body motion of particles are satisfied by adding a body force (fluid-solid interaction force) to the equation as follows:

$$
\begin{aligned}
\mathbf{f}_{H}\left(\mathbf{x}, t_{n}\right) & =-\phi\left(\mathbf{x}, t_{n}\right) \mathbf{f}_{P}\left(\mathbf{x}, t_{n}\right) \\
& =-\phi^{n}\left(\mathbf{u}_{P}\left(\mathbf{x}, t_{n}\right)-\mathbf{u}\left(\mathbf{x}, t_{n}\right)\right),
\end{aligned}
$$

where $\mathbf{u}_{P}\left(\mathbf{x}, t_{n}\right)$ and $\mathbf{u}\left(\mathbf{x}, t_{n}\right)$ are particle and fluid velocities at $t=t_{n}$, respectively. In LBM, there are several methods for introducing a body force term into the evolution equation. The most common method is adding a term into the collision operator as follows [8]:

$$
\begin{aligned}
& f_{\alpha}\left(\mathbf{x}+\mathbf{e}_{\alpha} \delta t, t_{n}+\delta t\right)=f_{\alpha}\left(\mathbf{x}, t_{n}\right) \\
& \quad-\frac{1}{\tau}\left[f_{\alpha}\left(\mathbf{x}, t_{n}\right)-f_{\alpha}^{\mathrm{eq}}\left(\mathbf{x}, t_{n}\right)\right]+\frac{\omega_{\alpha} \delta t}{c_{s}^{2}}\left(\mathbf{f}_{H} \cdot \mathbf{e}_{\alpha}\right) .
\end{aligned}
$$

It should be mentioned that this force is applied only to solid nodes in the computational domain. Outside the particle domain, this force is zero.

Hydrodynamic force, $\mathbf{F}_{i}^{H}$, and torque, $\mathbf{T}_{i}^{H}$, exerted on each solid particle are obtained from momentum conservation law as [10]:

$$
\begin{aligned}
& \mathbf{F}_{i}^{H}=\int_{\forall_{p_{i}}} \rho \phi^{n}\left(\mathbf{u}\left(\mathbf{x}, t_{n}\right)-\mathbf{u}_{p}\left(\mathbf{x}, t_{n}\right) d \forall_{p_{i}},\right. \\
& \mathbf{T}_{i}^{H}=\int_{\forall_{p_{i}}}\left(\mathbf{x}-\mathbf{R}_{i}^{n}\right) \times \rho \phi^{n}\left(\mathbf{u}\left(\mathbf{x}, t_{n}\right)-\mathbf{u}_{p}\left(\mathbf{x}, t_{n}\right) d \forall_{p_{i}} .\right.
\end{aligned}
$$

In these equations, $\forall_{p_{i}}$ is the $i$ th particle volume. The translational and angular velocities of each particle are updated using the following relations:

$$
\begin{aligned}
& \mathbf{V}_{i}^{n+1}=\mathbf{V}_{i}^{n}+M_{p_{i}}^{-1} \int_{t_{n}}^{t_{n}+\delta t}\left(\mathbf{F}_{i}^{H}+\mathbf{F}_{i}^{c}+\mathbf{F}_{i}^{\mathrm{ext}}\right) d s, \\
& \boldsymbol{\omega}_{i}^{n+1}=\boldsymbol{\omega}_{i}^{n}+\mathbf{I}_{p_{i}}^{-1} \int_{t_{n}}^{t_{n}+\delta t}\left(\mathbf{T}_{i}^{H}+\mathbf{T}_{i}^{\mathrm{ext}}\right) d s,
\end{aligned}
$$

where $M_{p_{i}}$ is the particle mass, $\mathbf{I}_{p_{i}}$ is the particle moment of inertia tensor, which is $I_{p_{i}}=\frac{1}{2} M_{p_{i}} R_{p_{i}}^{2}$ in our $2 \mathrm{D}$ simulation. $\mathbf{F}_{i}^{\text {ext }}$ and $\mathbf{T}_{i}^{\text {ext }}$ are external force and torques applied to the particle, and $\mathbf{F}_{i}^{c}$ is the collision force between the two particles in distances of the order of grid spacing.

Finally, the particle positions are updated by:

$$
\mathbf{R}_{i}^{n+1}=\mathbf{R}_{i}^{n}+\int_{t_{n}}^{t_{n}+\delta t} \mathbf{V}_{i} d s .
$$

\subsection{Particles near contact}

The forces exerted on particles can be resolved through the fluid-solid interaction force when the distance between the surfaces of the two particles contains enough fluid-computational nodes. When the gap width between the two particles is of the order of one lattice spacing, fluid-solid interaction force could not be effective, and chances are the particles cross each other. So, it is important to employ a numerical technique to prevent particles from penetrating into each other. To this end, a repulsive force is used in most cases. So, in the present work, a pair-wise repulsive force acting on the $i$ th particle due to its interaction with $j$ th particle is adopted. This extra short-range repulsive force is added as an external force into the total force that a particle experiences and has the following functional form [7]:

$$
\mathbf{F}_{i j}^{P}=\left\{\begin{array}{l}
0 \\
\quad\left|\mathbf{R}_{i}-\mathbf{R}_{j}\right| \succ 2 R_{p}+\zeta \\
\frac{c_{i j}}{\varepsilon_{p}}\left(\frac{\left|\mathbf{R}_{i}-\mathbf{R}_{j}\right|-2 R_{p}-\zeta}{\zeta}\right)^{2}\left(\frac{\mathbf{R}_{i}-\mathbf{R}_{j}}{\left|\mathbf{R}_{i}-\mathbf{R}_{j}\right|}\right) \\
\quad\left|\mathbf{R}_{i}-\mathbf{R}_{j}\right| \leq 2 R_{p}+\zeta
\end{array}\right.
$$

In this equation, $c_{i j}$ is a force scale and is set to be the buoyancy force in this study. $\mathbf{R}_{i}$ and $\mathbf{R}_{j}$ represent the particle positions, and $\zeta$ is the threshold or safe zone and is set to one lattice unit in this study. $\varepsilon_{p}$ is the stiffness parameter.

\section{Validation test and numerical results}

\subsection{Validation test}

The motion of a neutrally buoyant two-dimensional circular particle moving in a viscous shear flow is investigated to verify the present computational frame. A schematic representation of this problem is shown in Figure 3. Length and width of the simulated domain are $L_{x}=2000$ and $L_{y}=80$ expressed in lattice units, respectively. Particle diameter and kinematic viscosity are selected as 10 and $1 / 30$, respectively. Two parallel walls move in opposite directions with the same velocity, $U_{w} / 2=1 / 120$. Particle-based

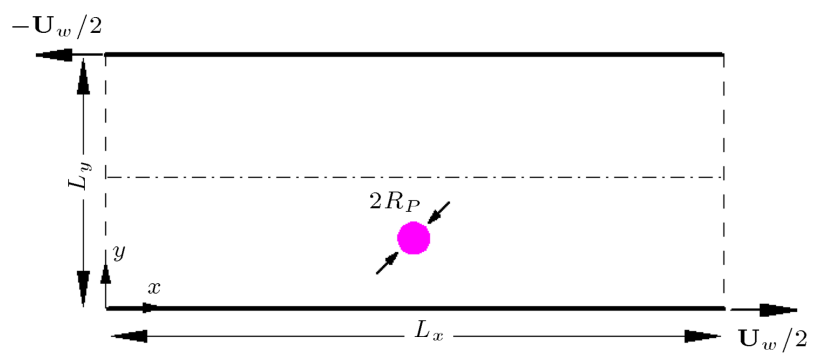

Figure 3. Schematic representation of a particle in shear flow. 


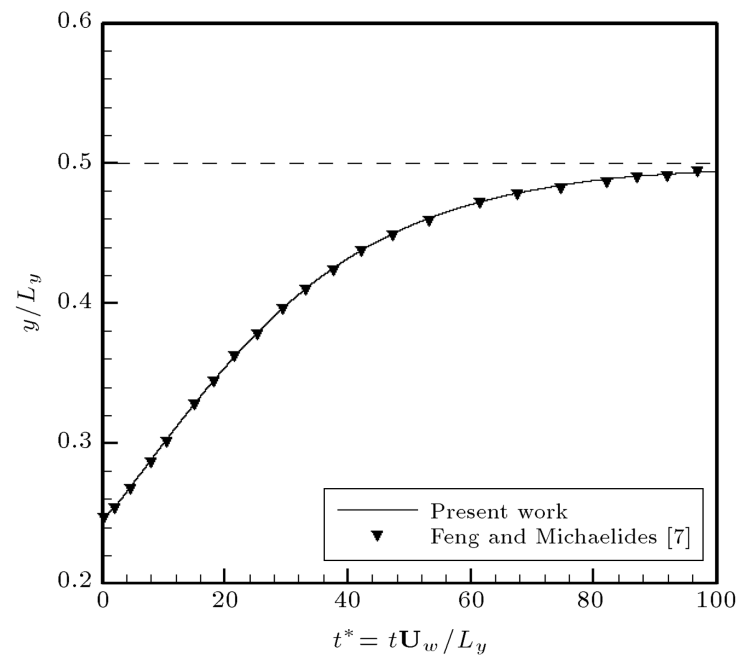

Figure 4. Non-dimensional transverse location of center of circular particle versus non-dimensional time.

Reynolds number is $\operatorname{Re}_{p}=\gamma d^{2} / v=0.625$, where the imposed shear rate $\gamma=1 / 4800$. The initial position of the particle is at $\left(L_{x} / 2, L_{y} / 4\right)$. Figure 4 shows the lateral migration of the particle in shear flow as compared with the numerical results of Feng and Michaelides [7]. Excellent correspondence is observed between the present computations and those of Feng and Michaelides [7].

\subsection{Numerical results}

Computational domain, which consists of a planar channel with stenosis, is shown in Figure 5. As is shown in this figure, length and width of the simulated domain are $L_{x}=32 d$ and $L_{y}=8 d$, where $d=8.5 \mu \mathrm{m}$ is the diameter of suspended particle. Two symmetric protuberances are used to create the stenosis inside the channel. The protuberances are semicircles of equal radius determined by the width of the stenosis throat $b$ which is selected as $d<b<2 d$. The center of these circular protuberances is located at the midpoint of the upper and lower walls. The suspended particles and fluid have the same density of $1 \mathrm{~g} / \mathrm{cm}^{3}$. Inlet and outlet boundary conditions are selected as specified pressures, which are implemented using the method of Zou and He [18]. Bounce-back boundary conditions are used at the upper and lower boundaries. Smoothed profile method is applied to satisfy the no-slip boundary condi-

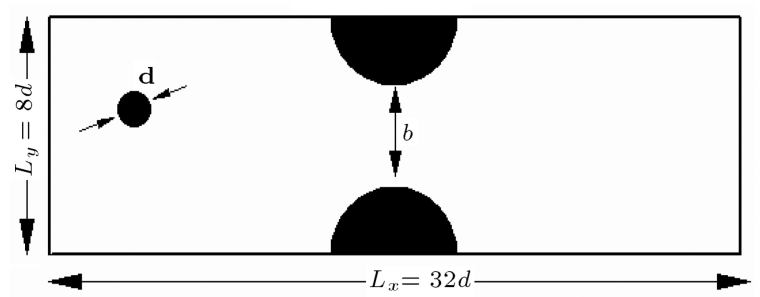

Figure 5. Schematic representation of the planar channel with stenosis. tion at protuberances and suspended particles surfaces. The numbers of lattice nodes and suspended particle diameter based on lattice units are selected as $448 \times 112$ and 14, respectively. Relaxation time is selected as $\tau=0.75$. Initially, computations were done for flow through channel with stenosis without any suspended particle and continued to reach its steady state after applying the known pressure difference. Figure 6 shows streamlines in the planar channel with two symmetric protuberances. The steady-state velocity field in this system is used as the initial velocity field for the following simulations.

Next, a suspended particle is placed initially at the centerline where its $x$-location of centerline is placed $2 d$ from the stenosis throat. In its subsequent motion, the center of particles stays at the centerline. Figure 7 shows $x$-component of velocity of center versus time at different pressure (density) differences. It is clear that speed of particle increases and reaches its maximum value at the stenosis throat, and then decreases after passing stenosis. As it is expected in such flow configurations, symmetric behavior is observed for velocity of particle with respect to the center of circular protuberances. It is also observed in Figure 7 that increasing pressure difference increases the maximum speed of the particle while decreasing the time spend for particle to arrive at the stenosis

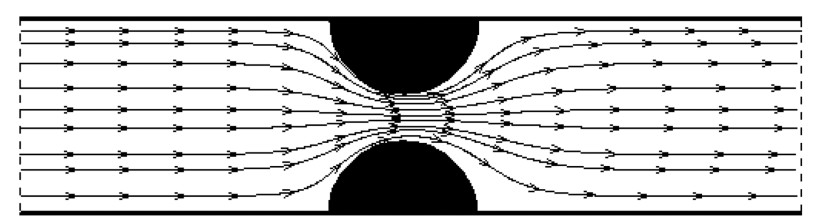

Figure 6. Streamlines in a channel with symmetric stenosis.

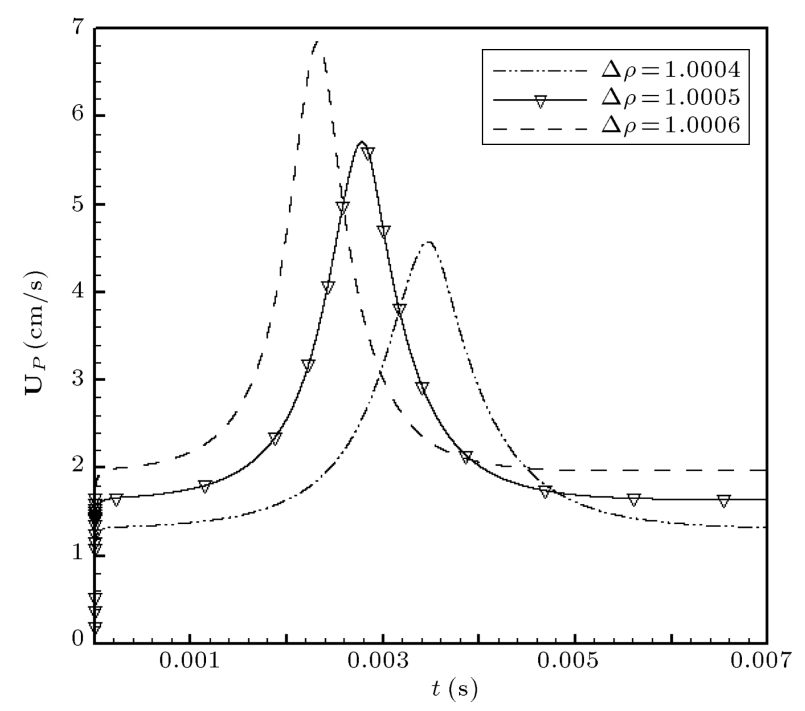

Figure 7. Effect of inlet and outlet pressure (density) difference on the $x$-component velocity of a particle placed at the centerline. 


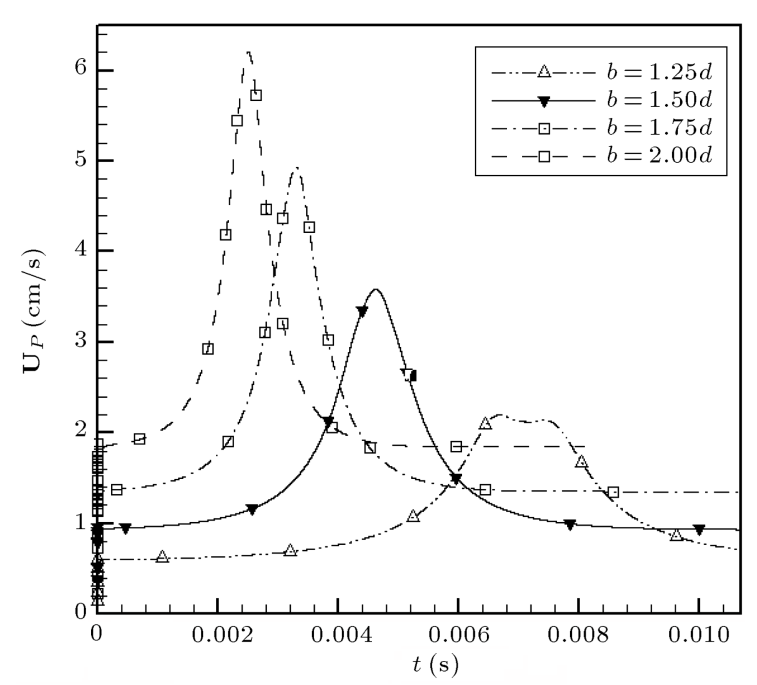

Figure 8. Effect of width of the stenosis throat on the $x$-component velocity of the particle.

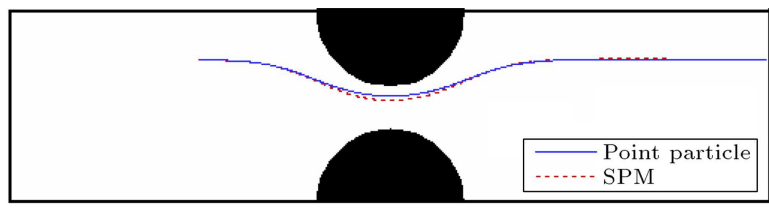

Figure 9. Comparison of the trajectory of the two particles released from the same initial position: A point particle and a particle having a volume comparable to the grid spacing (SPM).

throat. In addition to pressure difference between inlet and outlet boundary conditions, width of the stenosis throat also affects maximum speed while reducing elapsed time for passing a particle through the throat. In order to investigate such effect, particle's horizontal velocity at the centerline is shown in Figure 8. As is observed in this figure, increasing the width of the stenosis throat increases the maximum horizontal velocity of the particle.

In another simulation, a suspended particle is initially positioned $2 d$ above the centerline. The trajectory of the particle is shown in Figure 9. It is seen that the particle moves towards the centerline near and across the protuberance and comes back to its initial position after passing the stenosis throat. To show the importance of the present approach that accounts for the volume of the particle, the trajectory of a point particle which is released from the same initial position as the SPM particle is also shown in this figure. Comparison of two trajectories shows that the two particles track the same path before entering the stenosis throat. Just near the throat, the SPM particle deviates from the point particle. This is due to the repulsive force that the SPM particle experiences from the stenosis throat which pushes this particle towards the centerline, whereas the point particle has no effect derived from the throat.

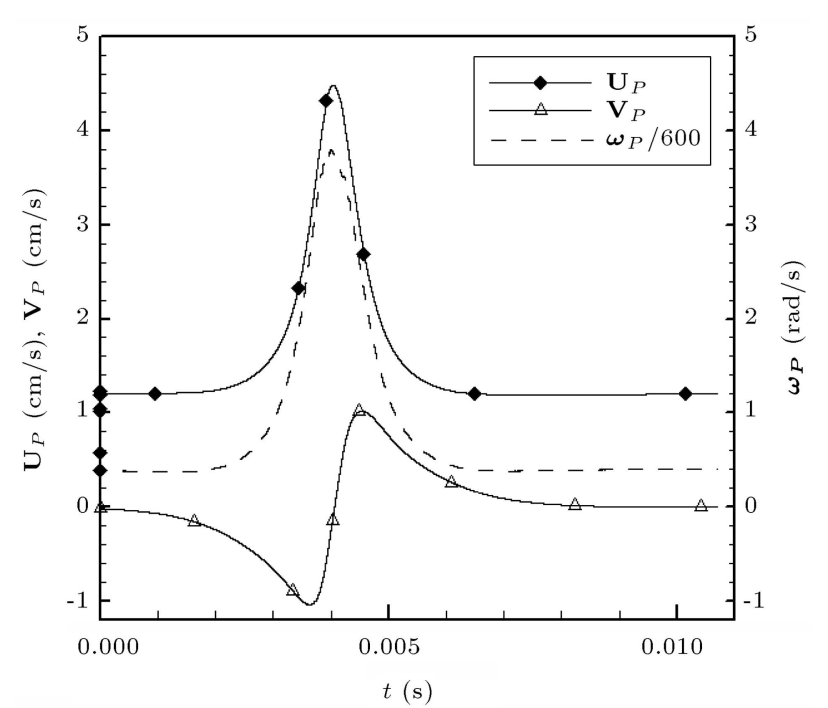

Figure 10. Time history of translational and angular velocities of a particle with the initial position above centerline.

Figure 10 depicts the instantaneous $x$ - and $y$ component of the velocity vector along with angular velocity of the particle. Horizontal velocity of particle starts increasing and reaches its maximum value at the throat with a symmetrical behavior after passing the throat. The vertical velocity of the particle remains close to zero except for an interval before and after the throat. In addition, as it is seen in this figure, angular velocity of particle at stenosis throat is not smooth. This is due to the exerted lubrication force between upper protuberance and the suspended particle.

Similar to the case of one particle, the movement of two circular particles in the channel has also been studied. Initial positions of these two articles are $(8 d, 2 d)$ and $(6.4 d, 6 d)$. Computational domain and particles diameter based on lattice units were selected as $448 \times 112$ and 14 , respectively. Particles start moving in the channel. Figures 11-13 depict time histories of the horizontal, vertical, and angular velocities of the two particles placed initially asymmetrical in the channel. It can be seen in Figure 11 that the maximum horizontal velocity of the second particle is a little smaller than that of the first one. This may be due to the placement of the first particle in front of the second one at the throat, and thus decreasing velocity of the second particle.

In order to investigate the effect of the shape of the stenosis on the resistance to particles' motion, a channel with two symmetric protuberances and six different shapes is considered. Figure 14 depicts these configurations in which the widths of the throat are $b_{1}=5 d, b_{2}=3.75 d, b_{3}=2.8 d, b_{4}=1.9 d, b_{5}=$ $1.5 d$, and $b_{6}=1.2 d$. This affects pressure difference and resistance to the flow. Figure 15 shows pressure difference variation in the channel versus width of 


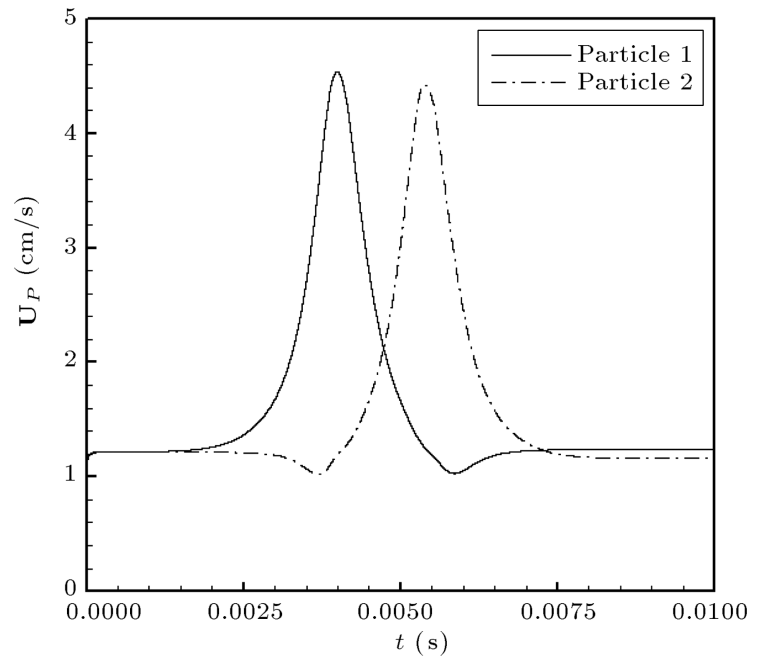

Figure 11. $x$-component of velocity of the two particles located asymmetrically in a planar channel with two symmetric protuberances.

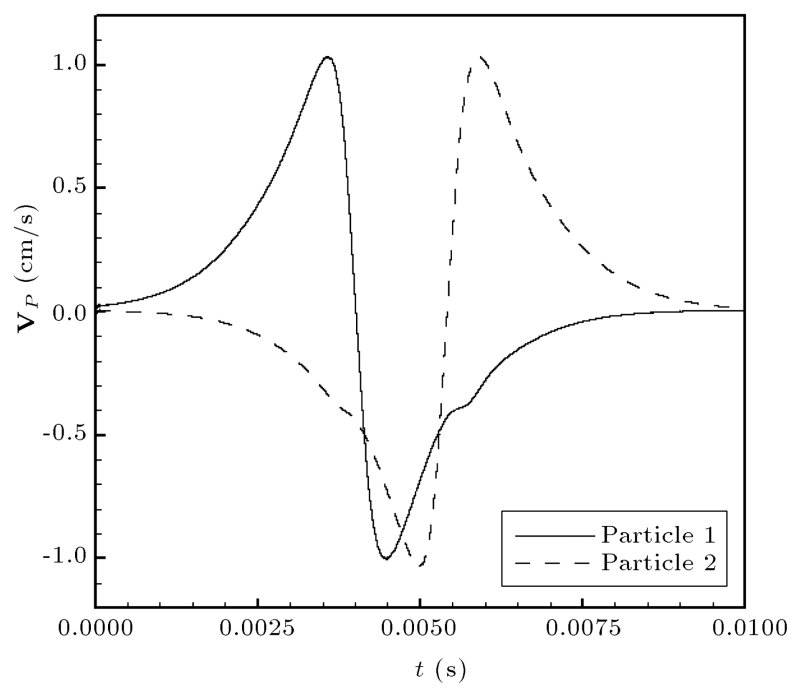

Figure 12. $y$-component velocity of the two particles located asymmetrically in a planar channel with two symmetric protuberances.

the stenosis in the mentioned configurations. To this end, the value of the pressure in one node before and after the protuberances is considered, and based on these nodes, the pressure difference is calculated. It is clear that pressure difference increases by increasing the height of protuberances or decreasing the width of stenosis.

Moreover, in order to find the effect of stenosis shape on particles' motion, two particles are placed asymmetrically in channel ahead of stenosis at positions $(8 d, 2 d)$ and $(6.4 d, 6 d)$. A schematic representation of this geometry is depicted in Figure 16. Boundary conditions in this investigation are uniform inlet velocity and outlet pressure. Figures 17-19 represent variation of the $x$ - and $y$-component of velocity

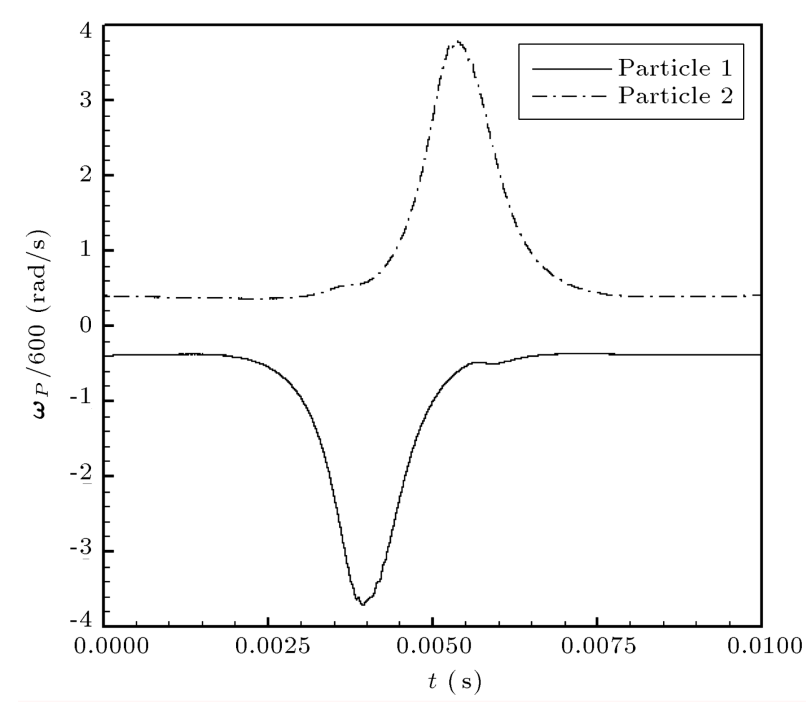

Figure 13. Angular velocity of the two particles located asymmetrically in a planar channel with two symmetric protuberances.

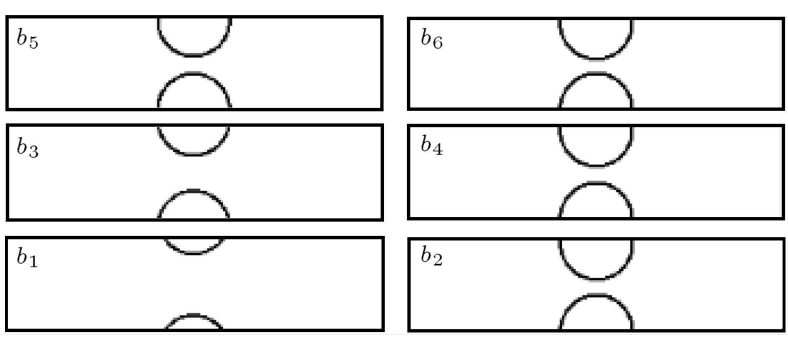

Figure 14. Schematic representation of six different shapes of the stenosis.

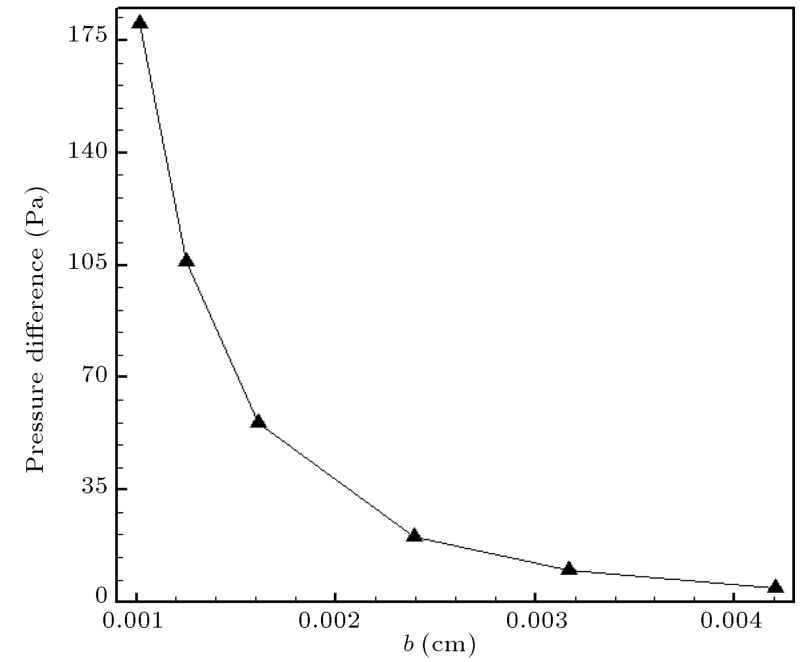

Figure 15. Pressure difference variation versus width of the stenosis in a channel with two symmetric protuberances for six configurations.

in addition to angular velocity of the first particle with time. It is observed that as the width of the stenosis decreases, translational and angular velocities of particle at stenosis increase which is due to the 


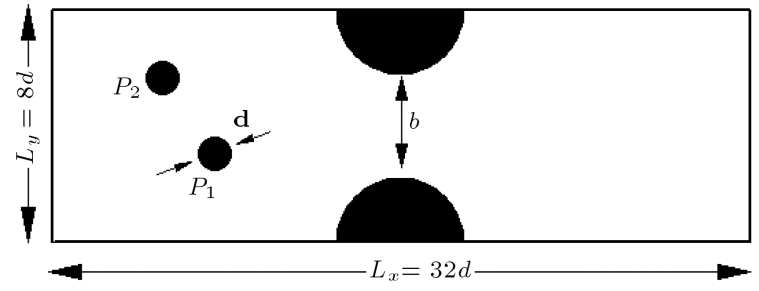

Figure 16. Schematic representation of the two particles in a planar channel with symmetric protuberances.

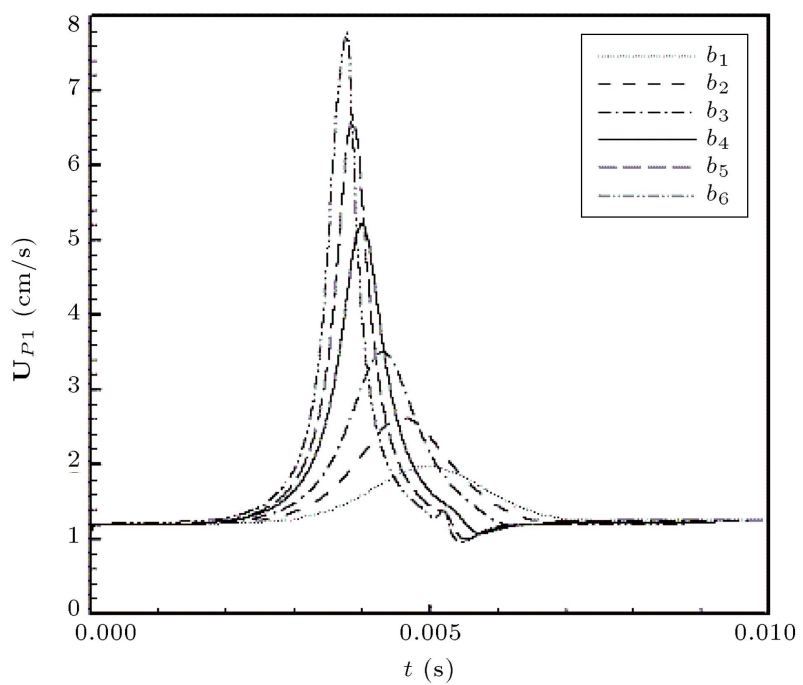

Figure 17. Time history of the $x$-component velocity of particle 1 at six configurations (particle 2 is also present in this simulation).

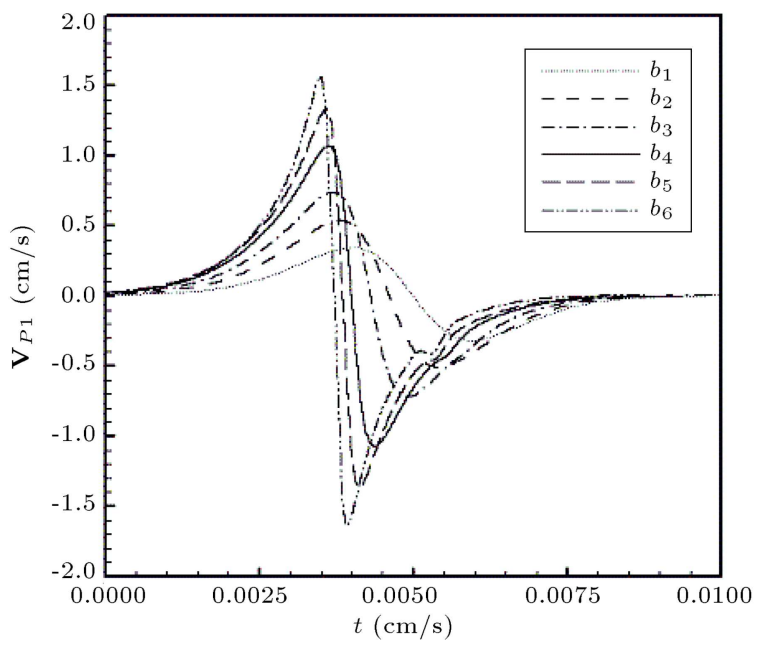

Figure 18. Time history of the $y$-component velocity of particle 1 at six configurations (particle 2 is also present in this simulation).

fact that fluid velocity at the stenosis increases by decreasing its width as well as particle which passes through it, should synchronize itself with fluid. Similar phenomenon happens to the second particle.

It is worth mentioning the simulation time in the present study. To this end, the simulation time

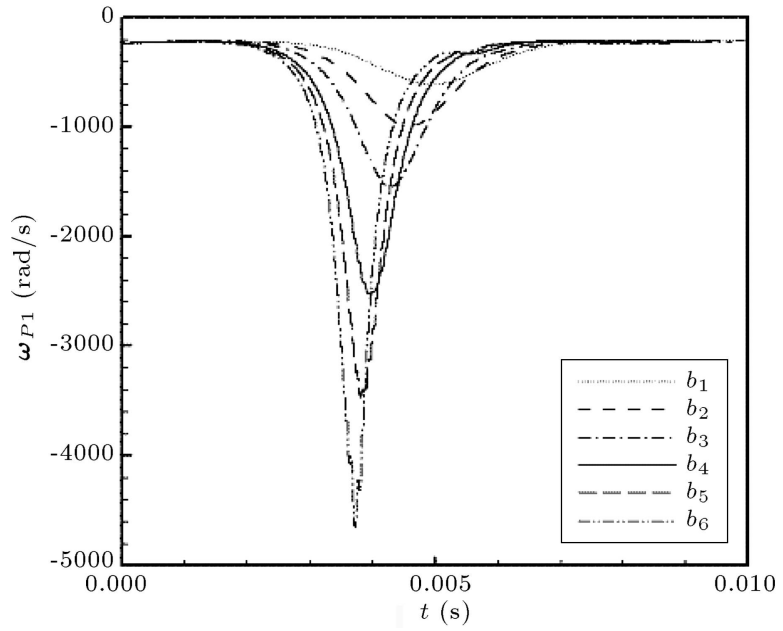

Figure 19. Time history of the angular velocity of particle 1 at six configurations (particle 2 is also present in this simulation).

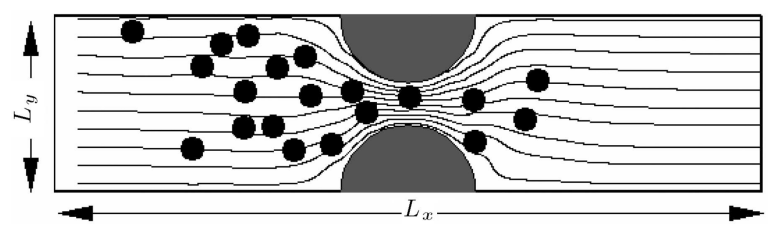

Figure 20. A snapshot of many particles in a symmetric stenosis together with the streamlines.

of three cases, namely a channel with symmetric protuberances, one particle at the centerline, and two particles positioned asymmetrically in a channel with symmetric protuberances, is tabulated in Table 1 .

Finally, in order to investigate the applicability of combined LBM-SPM for simulating many particles, a fluid flow containing twenty particles in the planar channel with two symmetric protuberances is also studied. Particles are distributed randomly in the channel in front of protuberances and start moving according to the imposed pressure difference. Snapshot of these particles along with streamlines is shown in Figure 20. Of prime importance in particulate flow is computational time which was about a few hours on a laptop computer for this configuration. It is concluded

Table 1. The simulation time in the present study at three simulated cases.

\begin{tabular}{lc}
\hline \multicolumn{1}{c}{$\begin{array}{c}\text { Simulated } \\
\text { case }\end{array}$} & $\begin{array}{c}\text { Simulation } \\
\text { time (s) }\end{array}$ \\
\hline A channel with symmetric & 836 \\
protuberances & 7502 \\
$\begin{array}{l}\text { One particle at the centerline of } \\
\text { a channel with symmetric protuberances } \\
\text { Two particles positioned asymmetrically in }\end{array}$ & 7854 \\
a channel with symmetric protuberances & \\
\hline
\end{tabular}


that such LBM-SPM is computationally efficient in predicting particulate flows.

\section{Conclusions}

The present work deals with simulation of fluid flow with one, two, and many particles in a planar channel with two symmetric protuberances. It was observed that horizontal velocity of particles increases in channel and reaches its maximum value at the throat. In addition, it was observed that angular velocity of particles was not smooth during particles motion, especially at throat. This is because of the lubrication force between protuberances and suspended particle which pushes particles away in the throat. Finally, a particulate flow with many particles in the channel was simulated which reveals the computational efficiency of combined LBMSPM in such flow configurations.

\section{References}

1. Srivastava, V.P. and Srivastava, R. "Particulate suspension blood flow through a narrow catheterized artery", Computers and Mathematics with Applications, 58, pp. 227-238 (2009).

2. Huabing, Li, Fang, H., Lin, Z., Xiong Xu, S. and Chen, S. "Lattice Boltzmann simulation on particle suspensions in a two-dimensional symmetric stenotic artery", Physical Review E, 69, p. 031919-1:9 (2004).

3. Wootton, D.M. and Ku, D.N. "Fluid mechanics of vascular systems, diseases, and thrombosis", Annu. Rev. Biomed. Eng., 01, pp. 299-329 (1999).

4. Aidun, C.K. and Clausen, J.R. "Lattice-Boltzmann method for complex flows", Annual Review of Fluid Mechanics, 42(1), pp. 439-472 (2010).

5. Ladd, A.J.C. "Numerical simulations of particulate suspensions via a discretized Boltzmann equation. Part I. Theoretical foundation", Journal of Fluid Mechanics, 271, pp. 285-310 (1994).

6. Ladd, A.J.C. "Numerical simulations of particulate suspensions via a discretized Boltzmann equation. Part II. Numerical results", Journal of Fluid Mechanics, 271, pp. 311-339 (1994).

7. Feng, Z. and Michaelides, E.E. "The immersed boundary-lattice Boltzmann method for solving fluidparticles interaction problems", Journal of Computational Physics, 195(2), pp. 602-628 (2004).

8. Mohammad, A.A. and Kuzmin, A. "A critical evaluation of force term in lattice Boltzmann method, natural convection problems", Int. J. Heat and Mass Transfer, 53, pp. 990-996 (2010).

9. Nakayama, Y. and Yamamoto, R. "Simulation method to resolve hydrodynamic interactions in colloidal dispersions", Physical Review E, 71(3), p. 036707-1:7 (2005).
10. Jafari, S., Yamamoto, R. and Rahnama, M. "LatticeBoltzmann method combined with smoothed-profile method for particulate suspensions", Physical Review E, 83(2), p. 026702-1:7 (2011).

11. Jahanshahi Javaran, E., Rahnama, M. and Jafari, S. "Particulate flow simulation using Lattice Boltzmann Method: A rheological study", Advanced Powder Technology, 25, pp. 1325-1333 (2014).

12. Filippova, O. and Hanel, D. "Lattice Boltzmann simulation of gas-particle flow in filters", Computers \& Fluids, 26, pp. 697-712 (1997).

13. Wu, J. and Shu, C. "Implicit velocity correction-based immersed boundary-lattice Boltzmann method and its applications", J. Comput. Phys., 228, pp. 1963-1979 (2009).

14. Shu, C., Liu, N.Y. and Chew, Y.T. "A novel immersed boundary velocity correction-lattice Boltzmann method and its application to simulate flow past a circular cylinder", J. Comput. Phys., 226, pp. 16071622 (2007).

15. Wu, J. and Shu, C. "Particulate flow simulation via a boundary condition-enforced immersed boundary Lattice Boltzmann scheme", Communications in Computational Physics, 7(4), pp. 793-812 (2009).

16. Succi, S., The Lattice Boltzmann Equation for Fluid Dynamics and Beyond, Oxford University Press, Oxford (2001).

17. Luo, X., Maxey, M.R. and Karniadakis, G.E. "Smoothed profile method for particulate flows: Error analysis and simulations", Journal of Computational Physics, 228(5), pp. 1750-1769 (2009).

18. Zou, Q. and He, X. "On pressure and velocity boundary conditions for the lattice Boltzmann BGK model", Phys. Fluids, 9, pp. 1591-1598 (1997).

\section{Biographies}

Akbar Kouhestani is a PhD student of Mechanical Engineering at Shahid Bahonar University of Kerman, Iran. He received his BSc degree from Amir Kabir university in 1994 and MSc degree from Tehran university in 1998. He is working on lattice Boltzmann simulation of particulate suspensions and also air conditioning systems.

Mohammad Rahnama is a Professor at Mechanical Engineering Department of Shahid Bahonar University of Kerman, Iran. He has been involved in fluid flow simulations, since 1997, the time he graduated from Shiraz University with a $\mathrm{PhD}$ degree. His interest in Lattice Boltzmann Method (LBM) as a strong simulation method in computational fluid dynamics started a few years ago with LBM simulation of a microchannel flow. He recently focused on investigation of solid particles motion immersed in a fluid flow using LBM which was a starting point for simulation of deformable 
particles. Now, he is continuing his research on motion and deformation of red blood cells in blood flow using LBM, FEM, and immersed boundary method.

Saeed Jafari received BS degree from Shahid Bahonar University of Kerman, MS degree from Isfahan University of Technology, and $\mathrm{PhD}$ degree from Shahid Bahonar University of Kerman, Iran in 2004, 2006, and 2010, respectively, all in Mechanical Engineering (Energy Conversion). He is currently an Assistant Professor of Mechanical Engineering at Shahid Bahonar
University of Kerman. His research interests include mathematical and numerical modeling, computational fluid dynamics, particle science and technology, simulation of multiphase flows, and Lattice Boltzmann method.

Ebrahim Jahanshahi Javaran is PhD of Mechanical Engineering from Shahid Bahonar University of Kerman. He is working on computational fluid dynamics with lattice Boltzmann method, desalination systems, solar energy and energy optimization. 